\title{
Optical Performance Monitoring from FIR Filter Coefficients in Coherent Receivers
}

\author{
F.N. Hauske ${ }^{1}$, J.C. Geyer ${ }^{2}$, M. Kuschnerov ${ }^{1}$, K. Piyawanno ${ }^{1}$, T. Duthel ${ }^{3}$, \\ C.R.S. Fludger ${ }^{3}$, D. van den Borne ${ }^{4}$, E.-D. Schmidt ${ }^{5}$, B. Spinnler ${ }^{5}$, \\ H. de Waardt ${ }^{4}$, B. Lankl ${ }^{1}$ \\ ${ }^{1}$ Federal Armed Forces University Munich, Institute for Communications Engineering, \\ D-85577 Neubiberg, Germany, e-mail: fabian.hauske@unibw.de \\ ${ }^{2}$ University of Erlangen-Nuremberg, Chair for High Frequency Technology, Cauerstr. 9, \\ D-91058 Erlangen, Germany, e-mail: jonas@coreoptics.com \\ ${ }^{3}$ CoreOptics, Nordostpark 12-14 D-90411, Nürnberg, Germany \\ ${ }^{4}$ COBRA institute, TU/e, Den Dolech 2, 5600 MB Eindhoven, The Netherlands \\ ${ }^{5}$ Nokia Siemens Networks GmbH \& Co. KG, Hofmannstr. 51, D-81379, Munich, Germany
}

\begin{abstract}
We present a robust and precise optical performance monitoring technique from FIR filter coefficients in coherent receivers with digital equalization. Residual chromatic dispersion, DGD and OSNR are simultaneously estimated from measured $111 \mathrm{Gbit} / \mathrm{s}$ data.
\end{abstract}

(C) 2008 Optical Society of America

OCIS codes: (060.1660) Coherent communications; (060.2360) Fiber optics links and subsystems

\section{Introduction}

As optical dense wavelength division multiplex (DWDM) networks become more flexible, optical performance monitoring (OPM) experiences increased importance. Key issues are residual chromatic dispersion (CD), differential group delay (DGD) and the optical signal-to-noise ratio (OSNR).

Various methods to monitor the signal are known. In general, they require cost-intensive external devices evaluating the optical spectrum. They also monitor the optical signal, neglecting filters and electric distortions that are crucial for the signal quality at the decision point. In addition, they tap the optical signal reducing the effective receive power [1] [2]. In [3] a state based OPM with simultaneous estimation of CD, OSNR and non-linearities was shown, which could be cost-effectively realized as a by-product in direct detection receivers with equalization by MLSE.

Recently an increased demand on bandwidth efficiency and flexible impairment mitigation lead to an intensified research in coherent demodulation ( [4], [5] and references therein). Complementary to direct detection, coherent demodulation gives a representative of the optical field in the electrical domain. This allows equalization by a complex valued FIR butterfly structure, where the filter coefficients relate to the inverse channel impulse response.

Within this work, we present a method to simultaneously estimate the parameters of CD, DGD and OSNR from FIR filter coefficients. The filters are blindly adapted based on measured data with off-line processing on a PC.

\section{Properties of the Impulse Response}

After the $90^{\circ}$ hybrid, the ADC and the clock recovery, we receive a digital representation of the receive signal (Fig. 1,

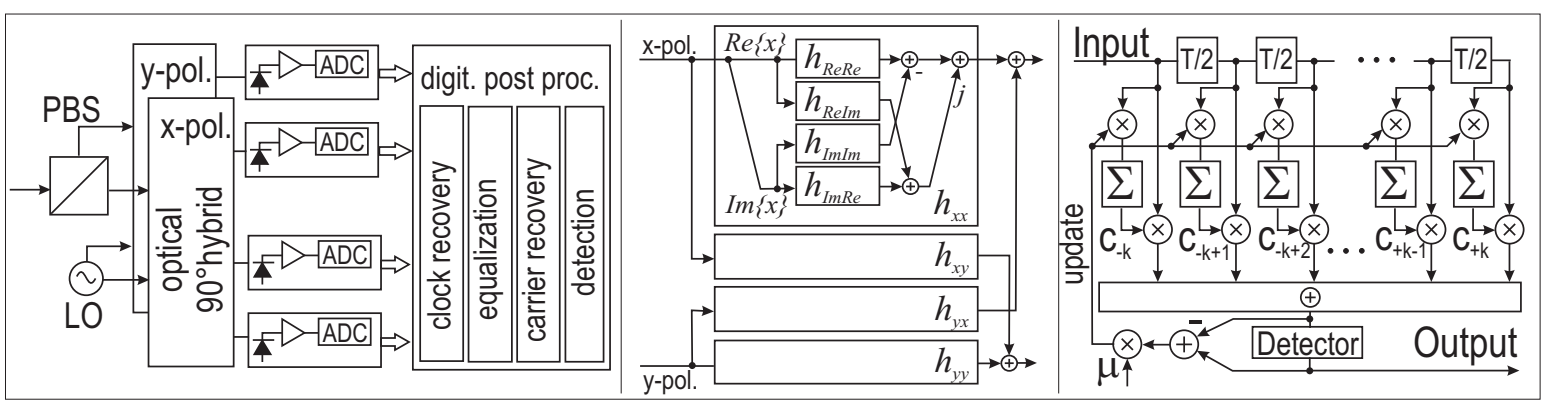

Fig. 1. Left: Block diagram of coherent receiver with digital equalization. Middle: Complex butterfly structure of the equalizer with exemplary detail of $h_{x x}$. Right: Tapped delay line of FIR filter with recursive LMS update of tap weight coefficients. 

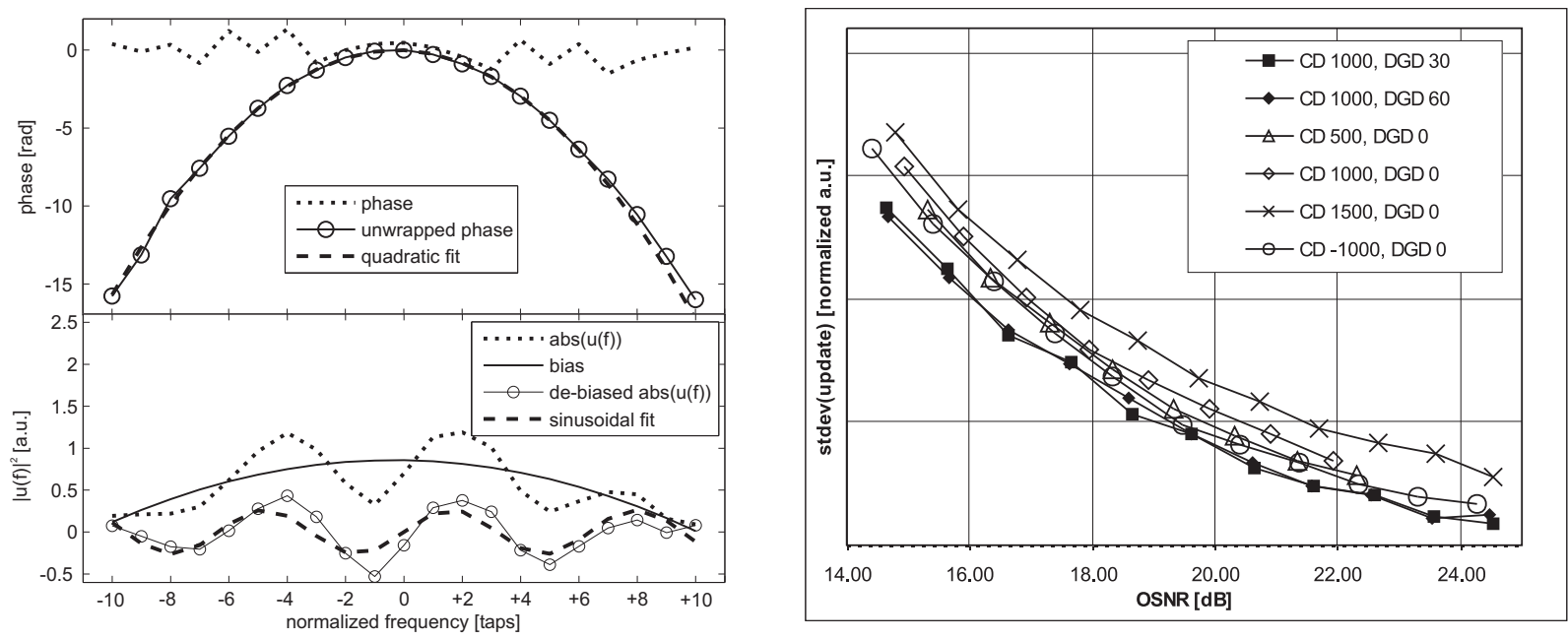

Fig. 2. Left: Parameter extraction from phase (top) and amplitude transfer function (bottom) for a given CD of $1000 \mathrm{ps} / \mathrm{nm}$ and DGD of $60 \mathrm{ps}$. Right: Correlation of OSNR and the variance in the update signal for several combinations of CD and DGD

left). Thus, in a real implementation we need a complex butterfly structure to compensate for PMD, where each filter block is realized by a real butterfly structure to represent the digital filter (Fig. 1, middle). The recursive LMS algorithm continuously updates the tap weight coefficients, which guarantees initial convergence and tracking of time variant channel distortions (Fig. 1, right). In steady state, the complex butterfly structure is a digital, real representation of the inverse impulse response defined by the tap coefficients.

To evaluate the filter properties we can make use of redundancy in the filter bank, to average on the coefficients and to obtain a more robust estimation. The coefficients of $h_{x x_{-} R e R e}$ and $h_{x x_{-} R e I m}$ should both contain information of the real part of $h_{x x}$. So we average on both of them to obtain the real part of $h_{x x}$. The same applies for $h_{x x_{-} I m R e}$ and $h_{x x_{-} I m I m}$ with respect to the imaginary part of $h_{x x}$.

If we transform the received inverse impulse response, obtained by blind convergence, into the frequency domain, we can easily derive the channel parameters for CD and DGD from the filter coefficients directly. The channel transfer function of the filter can be written as follows:

$$
\boldsymbol{H}^{-1}(f)=\boldsymbol{U}(f) \cdot D(f)=\left(\begin{array}{cc}
u(f) \cdot D(f) & v(f) \cdot D(f) \\
-v^{*}(f) \cdot D(f) & u^{*}(f) \cdot D(f)
\end{array}\right)
$$

where $\boldsymbol{U}(f)$ accounts for the inverse PMD matrix and $D(f)$ contains the inverse linear transfer function.

Operating in the linear regime, chromatic dispersion (CD) is the dominating effect. From Eq. 1 it becomes clear, that if we apply crosswise multiplication of the matrix elements

$$
u(f) D(f) \cdot u^{*}(f) D(f)-v(f) D(f) \cdot\left(-v(f)^{*}\right) D(f)=\left(|u(f)|^{2}+|v(f)|^{2}\right) D(f)^{2}=D(f)^{2}
$$

we can easily separate $D(f)$. The phase of $D(f)$ will follow a quadratic function, which makes it easy to unwrap the $2 \pi$-ambivalence [6]. With the aid of a quadratic fit we estimate the value of CD giving $\widehat{D}(f)$ (see Fig. 2 top).

Now we can eliminate the effect of CD from the transfer function by calculating $\boldsymbol{H}^{-1}(f) / \widehat{D}(f)=\widehat{\boldsymbol{U}}(f)$, which leads us to the inverse PMD matrix. It can be derived (not shown here) that the components of $\boldsymbol{U}(f)$ satisfy

$$
|u(f)|^{2}=(1+2 \gamma(1-\gamma) \cdot(\cos (2 \pi f \tau+\phi)-1))
$$

where $\gamma$ is the power splitting ratio, $\phi$ is an arbitrary phase shift between the PSPs, and $\tau$ is the DGD. The same applies for $v(f)$ with a negative sign. So after removing some bias, induced by the windowing effect of limited bandwidth of the filter, we can estimate the value of the DGD with a sinusoidal fit (see Fig. 2 bottom).

Finally the OSNR value can be estimated by the variance of the update signal.This applies to steady state operation, where the mean value of the update signal is constantly zero. Given a sufficient tap length, which is equal or longer than the channel memory length, the variance of the update signal becomes a function of the noise power of the received signal. With a reference measurement or normalization, the noise power or the OSNR can be estimated. 


\section{OThW2.pdf}
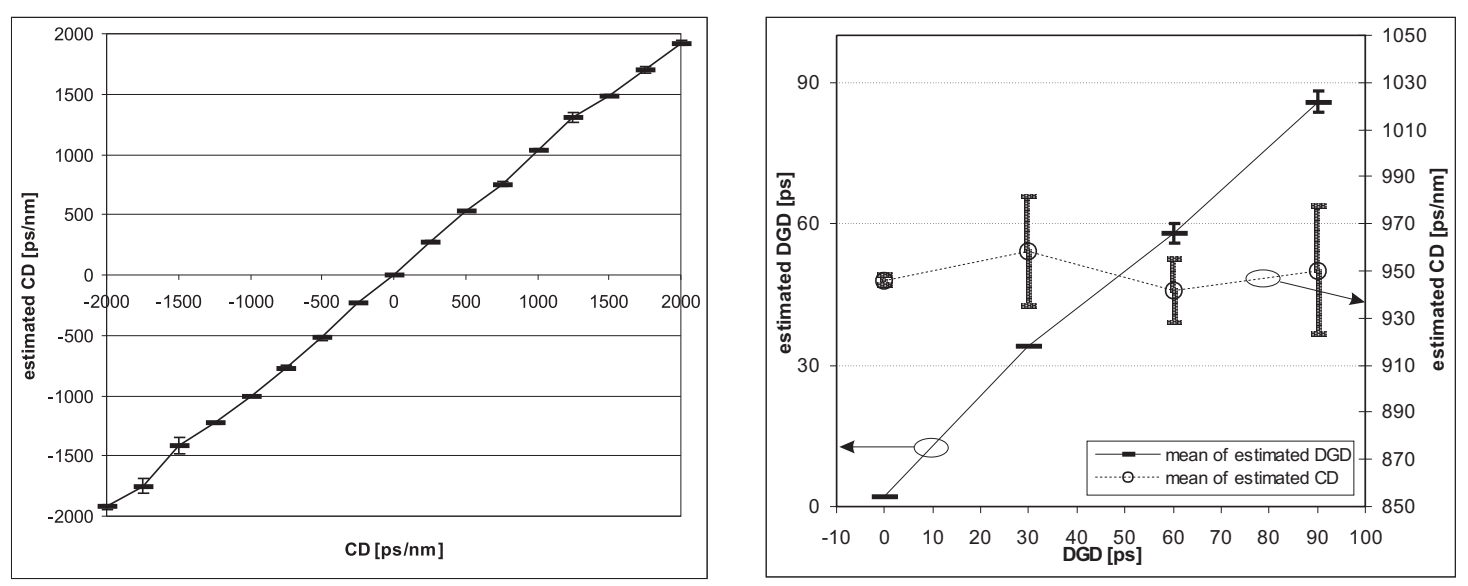

Fig. 3. Left: Estimated CD over a wide range of positive and negative chromatic dispersion with a very low standard deviation. Right: Estimation of combined CD and DGD with mean and standard deviation for each data point shown.

\section{Results from Measured Data}

Evaluations are based on measured $111 \mathrm{Gbit} / \mathrm{s}$ PolMUX-RZ-DQPSK data, stored in a digital sampling oscilloscope (2 samples/symbol) and processed off-line on a PC. After clock recovery the equalizer with 21, $T / 2$-spaced taps converged by blind adaptation. From the obtained filter coefficients, a fixed routine estimated all desired parameters.

Fig. 2, right, shows the relation of the variance of the update signal versus the OSNR. As long as the required channel memory is lower than the total filter length the estimation follows a monotonous function, which is nearly independent from the kind and the degree of the distortion. With a normalized abscissa the OSNR could be estimated with a precision of $\pm 1 \mathrm{~dB}$. In the case of $\mathrm{CD}=+1500 \mathrm{ps} / \mathrm{nm}$, the channel memory almost equals the filter length. So the update signal contains deterministic signal components, which can not be equalized for. In this case the function starts to be shifted by a certain offset. The offset even increases for higher values of CD.

The estimation of CD at zero DGD is given in Fig. 3, left. The estimation is accurate over a wide range from $-2000 \mathrm{ps} / \mathrm{nm}$ to $+2000 \mathrm{ps} / \mathrm{nm}$ in steps of $250 \mathrm{ps} / \mathrm{nm}$. For $\pm 2000 \mathrm{ps} / \mathrm{nm}$ the channel memory spreads over more than 12 symbol durations, which is longer than the filter length. Still the estimation delivers a precise value with a slight under-estimation. The highest standard deviation obtained for variations of the input OSNR from $12 \mathrm{~dB}$ to $24 \mathrm{~dB}$ was $\pm 63 \mathrm{ps} / \mathrm{nm}$ at $\mathrm{CD}=-1500 \mathrm{ps} / \mathrm{nm}$.

The combined effect of strong CD of $1000 \mathrm{ps} / \mathrm{nm}$ and DGD values of $0 \mathrm{ps}, 30 \mathrm{ps}, 60 \mathrm{ps}$ and $90 \mathrm{ps}$ is demonstrated in Fig. 3, right. The excellent estimation of the CD value, which is carried out first in the OPM routine, leads to a high accuracy of the DGD estimation. This proves, that the separation of both effects works well. Again, a low standard deviation for both parameters for variations of OSNR from $12 \mathrm{~dB}$ to $24 \mathrm{~dB}$ is shown. Equally good results were obtained from filters with 11 and 31 taps.

\section{Conclusion}

We have shown a practical approach for independently estimating the parameters of CD, DGD and OSNR in a coherent receiver for $111 \mathrm{Gbit} / \mathrm{s}$ PolMUX-RZ-DQPSK with digital filter based equalization. The estimation proved to be accurate and robust even in presence of combined distortions. The implementation with low computation effort comes as a by-product of the equalizer and could be cost effectively realized as part of coherent receivers.

\section{References}

1. D.C. Kilper et al., "Optical Performance Monitoring”, JLT 2004, vol. 22, no.1, p.294.

2. A. Kirstaedter et al., "Current Aspects of optical performance monitoring and failure root cause analysis in optical WDM networks", APOC 2004.

3. F.N. Hauske et al., "Optical Performance Monitoring in Amplitude Sampling Receivers", OECC2007, 12B4-3

4. C.R.S Fludger et al., "10 x 111 Gbit/s, 50 GHz Spaced, POLMUX-RZ-DQPSK Transmission over 2375 km Employing Coherent Equalization", OFC2007, PDP22

5. T. Duthel et al., "Impariment Tolerance of $111 \mathrm{Gbit} / \mathrm{s}$ PolmMUX-RZ-DQPSk using a reduced Complexity Coherent Receiver with a Tspaced Equalizer", ECOC2007, Mo1.3.2

6. G.P. Agrawal, Nonlinear Fiber Optics (Academic Press, 2001), Chap. 1.2. 